\title{
Laser Raman spectra and free and restricted rotation in phenyl silicates
}

\author{
M N DIXIT, N S K PRASAD and V B KARTHA* \\ Bhabha Atomic Research Centre, Bombay 400085, India
}

\begin{abstract}
Tetraphenyl silicate and related phenyl-alkyl silicates, an interesting class of compounds with applications in high vacuum technology, belong to the class of non-rigid molecules where free or restricted internal rotation of the substituent phenyl and alkyl groups provide several possible relative orientations. This may result in the formation of different phases under different conditions, providing results of fundamental interest in molecular dynamics. We have recently studied the laser Raman and infrared spectra of some of these compounds including tetraphenyl, phenyl triphenoxy, alkyl triphenyl, diphenyl dialkyl, phenyl trialkyl, and tetra alkyl silicates. Changes are observed in the spectra of these molecules with temperature, and these indicate the onset of restricted rotation at lower temperatures. These spectral changes and assignments are discussed in the paper.
\end{abstract}

Keywords. Free and restricted rotation; phenyl silicates; laser Raman spectra; non-rigid molecules.

\section{Introduction}

Tetraphenyl silicate and related phenyl-alkyl silicates form an interesting class of compounds with applications in high vacuum technology.

These compounds belong to the class of non-rigid molecules where free or restricted internal rotation of the substituent phenyl and alkyl groups provide several possible relative orientations. This may result in the formation of different phases under different conditions, providing results of fundamental interest in molecular dynamics. No spectral information is available for these compounds at present. The laser Raman and IR spectra are presented and discussed here for the first time.

\section{Experimental}

All the compounds were synthesised in our laboratories (N S K Prasad and K S Iyer, unpublished) and purified by repeated vacuum distillation until IR spectra showed the absence of all impurities.

IR spectra were obtained on a Model 180 Perkin-Elmer IR spectrometer. Raman spectra were obtained on a Spex Ramalog Raman Spectrometer which has been interfaced with a Wipro-PC computer (Ravindranath 1988). Excitation was carried out by 514.5 and $488.0 \mathrm{~nm}$ lines of a Spectra Physics $\mathrm{Ar}^{+}$laser. Powers from 50 to $400 \mathrm{~mW}$ were employed at the sample. Samples were contained in capillaries. Spectral slitwidths of 1 to $2 \mathrm{~cm}^{-1}$ were used for both IR and Raman recordings.

\footnotetext{
* For correspondence
} 


\section{Results and discussion}

The IR and laser Raman spectra of tetra phenyl silicate (TPS), phenyl triphenoxy silicate (PTPS) and alkyl triphenoxy silicate (ATPS) in the regions of interest in the present work, are shown in figures 1 to 5 . Figures 1 and 2 show the IR spectra of PTPS and TPS in the region $250-600 \mathrm{~cm}^{-1}$ and $600-1600 \mathrm{~cm}^{-1}$. Figure 3 shows the IR

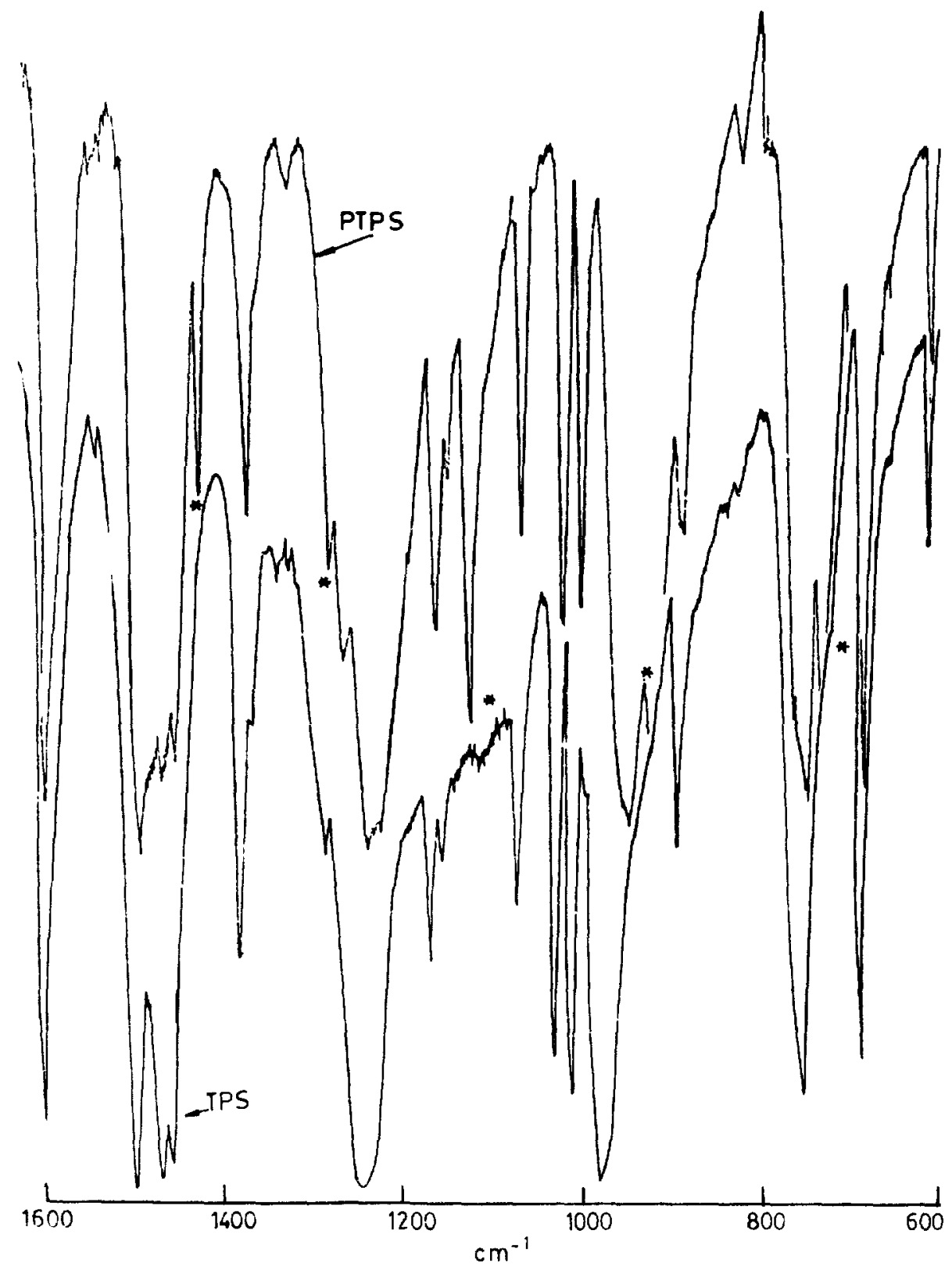

Figure 1. Infrared spectra of tetraphenyl silicate (TPS) and phenyl triphenoxy silicate (PTPS). The bands marked with an asterisk are due to the $\mathrm{C}_{6} \mathrm{H}_{5}$ group in PTPS, which is different from the other three $\mathrm{C}_{6} \mathrm{H}_{5}$ groups that are linked through oxygen. 


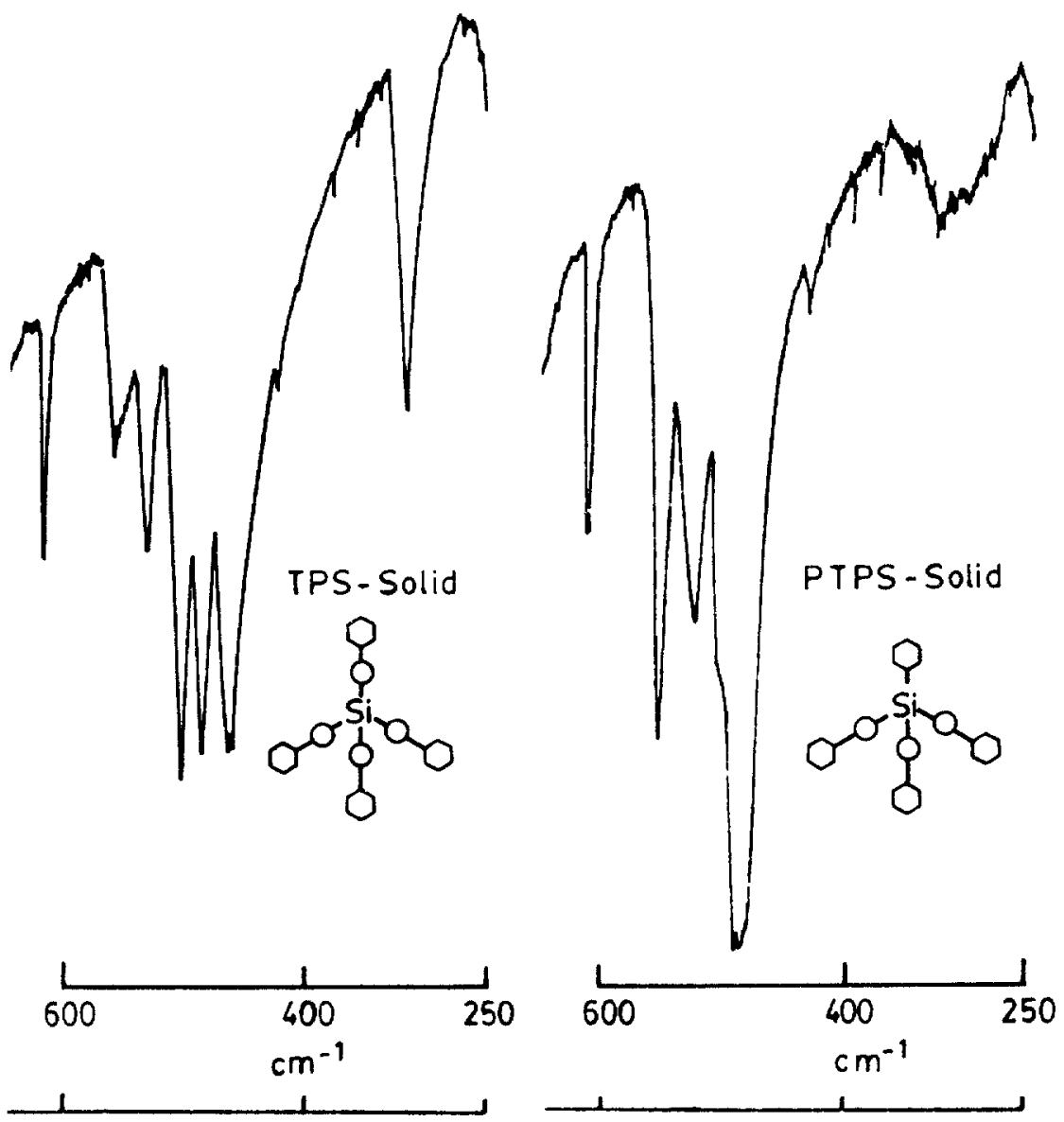

Figure 2. IR spectra of TPS and PTPS in the $600-300 \mathrm{~cm}^{-1}$ region, showing the various modes arising from restricted rotation (torsion) around the $\mathrm{Si}-\mathrm{O}$ and $\mathrm{O}-\mathrm{C}$ bonds.

spectra of ATPS in liquid form, and PTPS and TPS in solution form. Laser Raman spectra of TPS in melt and in solid forms are compared in figure 4. Figure 5 shows the spectrum of TPS as compared to its spectrum in benzene.

Since these molecules are quite big, a complete assignment of the frequencies corresponding to all the fundamental vibration modes is very difficult. Considering the typical case of TPS - tetraphenyl silicate $\mathrm{Si}\left(\mathrm{OC}_{6} \mathrm{H}_{5}\right)_{4}^{\circ}$ has a total of 141 fundamental modes, which can be classified as below:

\begin{tabular}{lc} 
Ring in-plane vibrations & $21 \times 4$ \\
Ring out-of-plane vibrations & $9 \times 4$ \\
Si-O stretch & 4 \\
O-Si-O bend & 5 \\
Si-O-C bend & 4 \\
Phenyl ring torsion & 4 \\
Phenoxy torsion & 4 \\
\hline & 141
\end{tabular}




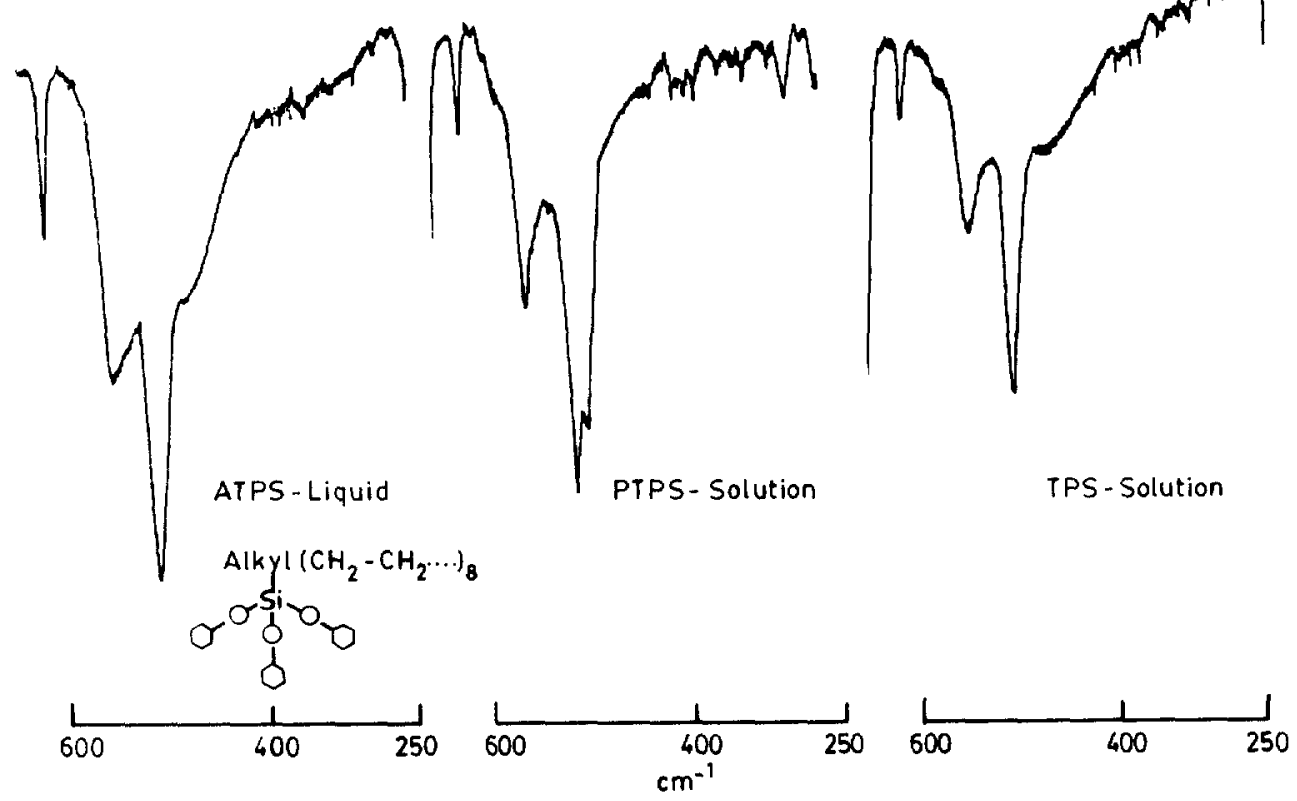

Figure 3. IR spectra of TPS, alkyl triphenyl silicate (ATPS) and PTPS, in the liquid or solution state, in the $600-300 \mathrm{~cm}^{-1}$ region. All the bands in figure 2, arising from restricted rotation are absent, leaving only ring torsion.

Assuming free rotation and $T_{d}$ symmetry these vibrations could be assigned to $12 \mathrm{a}$, $12 \mathrm{e}$ which are only Raman active, $11 \mathrm{f}_{1}$ which are inactive, and $24 \mathrm{f}_{2}$ which are both IR and Raman active.

The assignments of the various modes which are of interest from the point of view of free and restricted rotation, are shown in table 1 . The other assignments also can be made easily by comparison among the various compounds, as well as by comparison with spectra of substituted benzenes (Varsanyi and Szoke 1969).

The change of symmetry from tetra phenyl $\rightarrow$ triphenoxy, phenyl silicate $\rightarrow$ triphenoxy alkyl silicate makes it very easy to identify the various group frequencies. To almost every band in the tetraphenyl silicate (TPS) an additional band is introduced in the PTPS, because of the phenyl group being different from the phenoxy group.

The most interesting parts of the spectra are in the $400-700 \mathrm{~cm}^{-1}$ region. In solid TPS and PTPS several sharp peaks are observed in this region. These have to be assigned to the various torsional modes which include the 'ring out-of-plane' modes as well as the various torsional modes shown in table 1 . While the ring out-of-plane modes should be present in all phases, the torsional modes may exist only in solid phase, where the rotational motions are restricted. In solutions or in melts, free rotation sets in and these modes should broaden or disappear. This is clear from figure 3, where the bands at 316,480 and $530 \mathrm{~cm}^{-1}$ disappear in solution and in melt, while the bands at 615 and $680 \mathrm{~cm}^{-1}$ remain unchanged.

In conclusion, vibrational modes corresponding to the phenoxy and phenyl torsions of the phenyl silicates, having restricted motion in solid, clearly exhibit free rotation in solution or melt. 


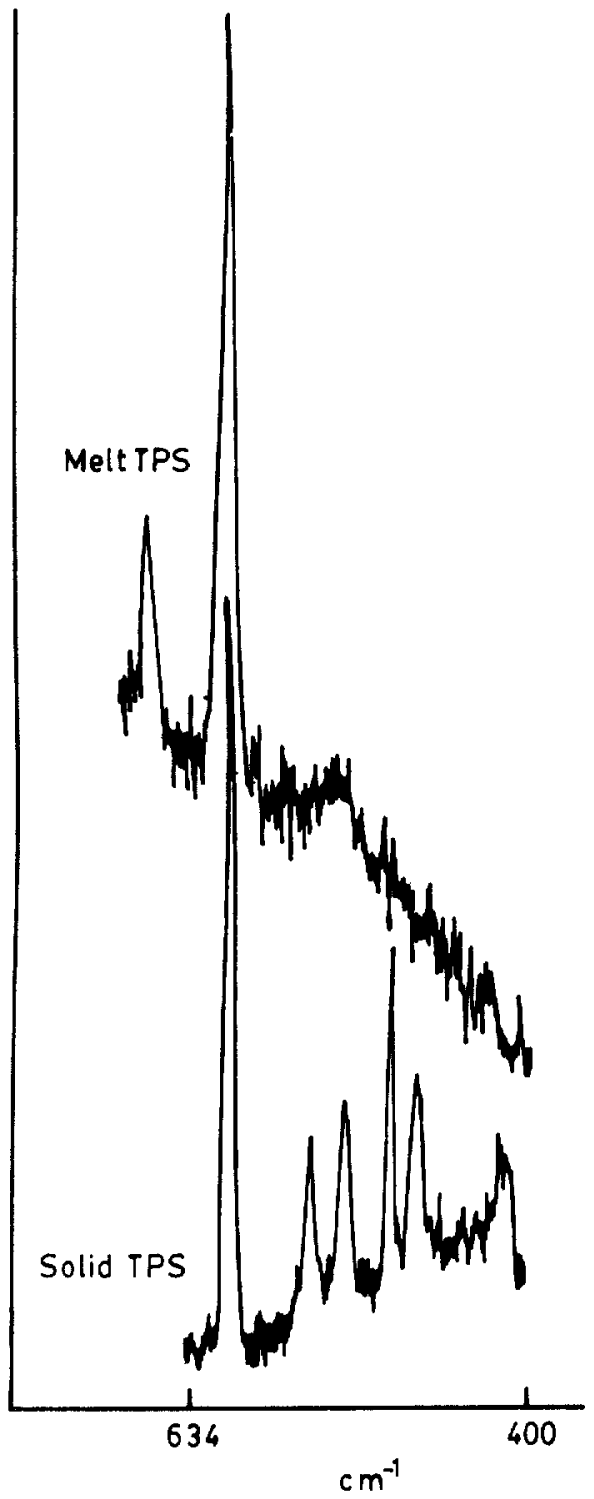

Figure 4. Raman spectra of TPS in solid and melt. The bands due to restricted rotation in solid are absent in the melt, only ring modes being left. 


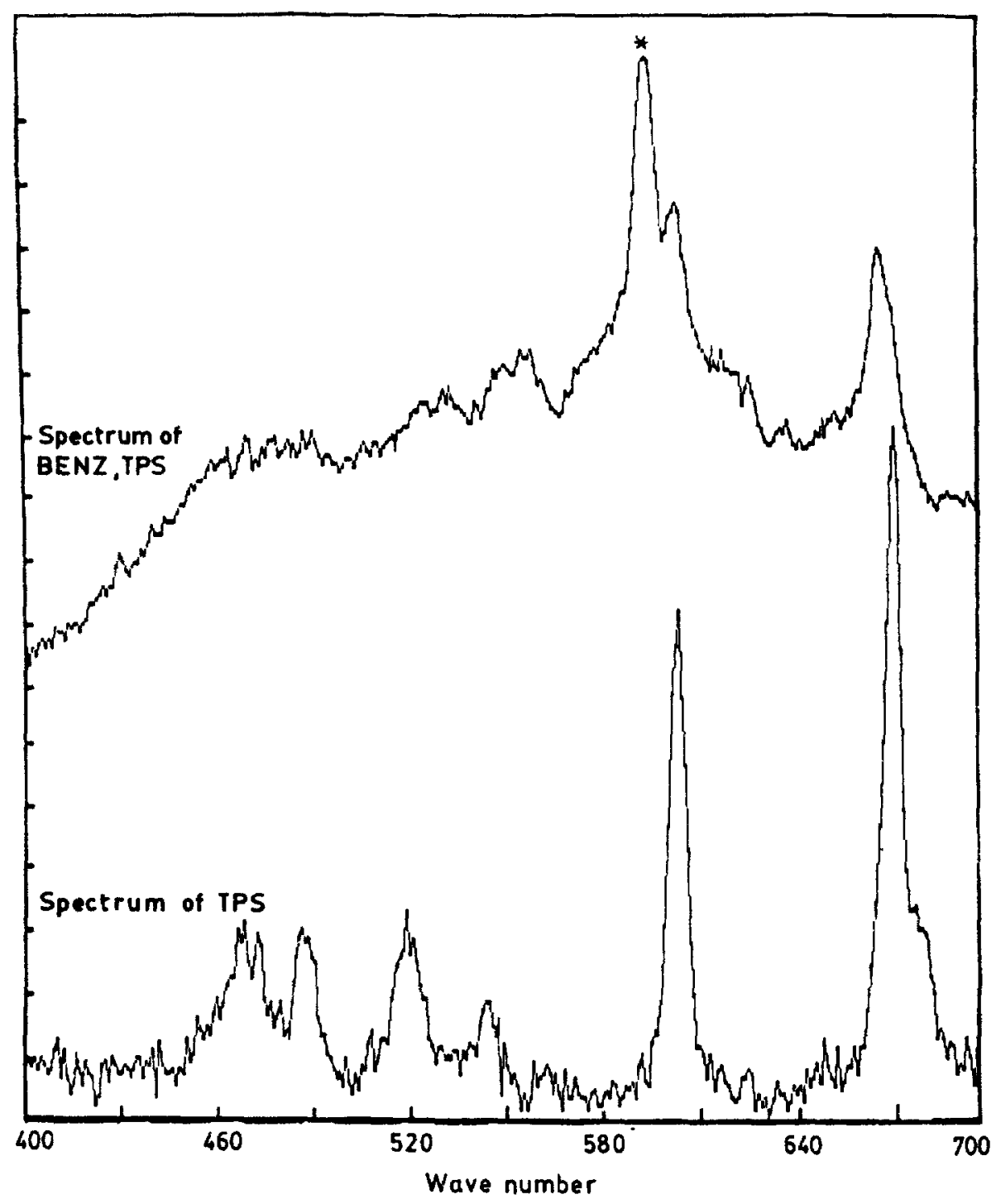

Figure 5. Raman spectra of TPS in solid and solution, showing disappearance of restricted rotation modes in solution. 
Table 1. Frequencies and assignments of some IR some IR and laser raman bands of phenyl silicates.

\begin{tabular}{|c|c|c|c|c|c|c|}
\hline \multicolumn{2}{|c|}{$\begin{array}{l}\text { Tetra phenyl } \\
\text { silicate }\end{array}$} & \multicolumn{2}{|c|}{$\begin{array}{l}\text { Phenyl triphenoxy } \\
\text { silicate }\end{array}$} & \multicolumn{2}{|c|}{$\begin{array}{l}\text { Alkyl triphenoxy } \\
\text { silicate }\end{array}$} & \multirow[b]{2}{*}{ Assignments* } \\
\hline Raman & IR & Raman & IR & Raman & IR & \\
\hline $316 w$ & $314 m$ & - & $320 w b$ & -- & - & Torsion \\
\hline - & $460 \mathrm{~ms}$ & - & $460 v w, v b$ & $470 w$ & $475 v b$ & Torsion \\
\hline $479 m w$ & $483 \mathrm{~ms}$ & $490 v w$ & $485 \mathrm{~s}$ & - & - & Torsion \\
\hline $499 m$ & $498 \mathrm{~ms}$ & $506 v w$ & $503 s$ & $502 m$ & $504 \mathrm{~ms}$ & $v_{16 a}$ \\
\hline- & - & - & $525 s$ & - & -- & Ring $v_{16}$ \\
\hline $532 \mathrm{mw}$ & $528 \mathrm{~m}$ & $533 v w$ & sh & $529 m$ & $525 s h$ & Torsion \\
\hline $557 w$ & $554 m$ & $555 v w$ & $556 s$ & $544 w$ & $545 m$ & $v_{16 b}$ \\
\hline $614 s$ & $615 m$ & $\begin{array}{l}614 s \\
619 s h\end{array}$ & $615 s$ & $616 s$ & $615 m$ & $v_{6 b}$ \\
\hline $681 \mathrm{~s}$ & - & 677 us & - & $678 v s$ & - & $v_{6 a}$ \\
\hline $690 w$ & $688 v s$ & $\sim$ & $\begin{array}{l}690 \mathrm{vs} \\
700 \mathrm{vs}\end{array}$ & - & $689 v s$ & $v_{4}$ \\
\hline $753 s$ & $755 v s$ & 758 & $753 v s$ & $755 m$ & $758 v s$ & $\gamma-\mathbf{C H}$ \\
\hline
\end{tabular}

*The bands assigned to torsion disappear on melting or in solution as seen in figures 3 and 4

Abbreviations: $b$-broad; $m$-medium; $s$-strong; sh-shoulder; $v$-very; $w$-weak.

\section{References}

Ravindranath S V G 1988 Indian Society of Analytical Scientists Natl. symp. on Analytical Spectroscopy Including Hyphenated Techniques, Hyderabad

Varsanyi G and Szoke S 1969 Vibrational spectra of benzene derivatives (New York: Academic Press) 